\title{
Factores en la asimilación de los fundamentos del Diseño Gráfico
}

\section{Factors in the assimilation of the Fundamentals of Graphic Design}

\author{
RIPALDA, Daniel ${ }^{1}$ \\ GALLAR, Yamirlis ${ }^{2}$ \\ ORMAZA, César D. ${ }^{3}$
}

\begin{abstract}
Resumen
El estudio evaluó la asimilación de los fundamentos del Diseño Gráfico en el proceso de enseñanza aprendizaje; el objetivo del trabajo estuvo dirigido a determinar los factores que inciden en la formación de competencias relacionadas a la composición de piezas visuales, para lo cual se aplicaron distintos métodos de recolección e interpretación de información, determinando diversos problemas metodológicos en varias instituciones de Educación Superior del Ecuador, en las que se ofertan Carreras relacionadas con el Diseño Gráfico. Los datos obtenidos en este trabajo presentan indicios de la existencia de inconvenientes en la formación de competencias básicas en los diseñadores y artistas visuales de cinco de las escuelas más importantes de Diseño del Distrito Metropolitano de Quito en la República del Ecuador.
\end{abstract}

Palabras clave: diseño, fundamentos, gestalt, enseñanza

\begin{abstract}
The study evaluated the assimilation of the foundations of Graphic Design in the teaching-learning process; the objective of the work was to determine the factors that influence the formation of competences related to the composition of visual pieces, for which different methods of collecting and interpreting information were applied, determining various methodological problems in several institutions of Higher Education in Ecuador, in which Careers related to Graphic Design are offered. The data obtained in this work present indications of the existence of inconveniences in the formation of basic skills in designers and visual artists of five of the most important Design schools of the Metropolitan District of Quito in the Republic of Ecuador.

Key words: design, fundamentals, gestalt, teaching
\end{abstract}

\section{Introducción}

El diseño gráfico es una disciplina que permite comunicar mensajes a grupos específicos, a través de distintos recursos y soportes visuales, multimedia e hipermedia. Sus inicios como profesión datan de finales del siglo IX y sus fundamentos se remontan a la creación de la Bauhaus en 1919, desde la cual se cimentaron los principios del Diseño Industrial y el Diseño Gráfico, incorporando conceptos de las artes plásticas, psicología, arquitectura, fotografía, entre otras áreas del conocimiento.

\footnotetext{
${ }^{1}$ Coordinador. Carrera de Diseño Digital y Multimedia. Universidad Indoamérica. danielripalda@uti.edu.ec

${ }^{2}$ Coordinadora. Departamento Pedagógico. Universidad Internacional SEK. yamirlis.gallar@uisek.edu.ec

${ }^{3}$ Estudiante. Carrera de Diseño Digital y Multimedia. Universidad Indoamérica. dayndavid@gmail.com
} 
Esta profesión en constante evolución ha ido diversificándose e interviniendo en prácticamente todos los ámbitos de la producción de bienes y servicios a nivel mundial; actualmente tiene relación directa con los ámbitos editoriales, publicidad, marketing, informática y el Social Media Managemet; áreas que se han servido del Diseño gráfico para la generación, producción y difusión de mensajes con fines comerciales.

La formación de diseñadores gráficos profesionales contempla la adquisición de distintas competencias básicas y específicas, que se sustentan en la asimilación de los fundamentos del Diseño, principios constantes que se adquieren durante los primeros años de formación y que se relacionan con los distintos ámbitos del ejercicio profesional al margen de la especialidad que todo diseñador adquiere durante la Educación Superior.

Los fundamentos del Diseño han variado poco, a partir de la obra clásica de Wucius Wong (Wong, 1989) y se actualizaron a los nuevos soportes y software (Lupton \& Phillips, 2009); sin embargo, en ambas obras se coincide en que la forma, color, composición y la Gestalt, son conceptos esenciales en la ejecución de cualquier propuesta de diseño, sea editorial, publicitario, tipográfico, corporativo o señalético.

\section{Metodología}

\subsection{Primeros síntomas de un problema}

En la Carrera de Diseño Digital y Multimedia de la Universidad Indoamérica de Ecuador, en su Sede Quito, los docentes fueron notando algunas falencias en los proyectos finales de ciertas asignaturas en distintos periodos. Estas falencias a menudo sutiles y en otras ocasiones evidentes, apuntaban a que existía fallas en la asimilación de algunos conceptos relacionados a los procesos de composición de distintas piezas visuales realizadas por los estudiantes.

En distintos grupos académicos y reuniones de trabajo se discutieron las razones que podrían haber provocado estos inconvenientes por lo que se verificaron rigurosamente algunos indicadores de gestión docente; se analizó la planificación micro curricular de la Carrera de Diseño Gráfico, determinando que en gran medida los contenidos de las asignaturas de formación básica cumplen con lo planteado en el proyecto de la Carrera; incluso hay asignaturas que sobrepasan los contenidos esenciales y acorde a las actas de verificación del comité curricular, la cantidad de contenidos repetitivos o poco relevantes es marginal.

Durante el periodo 2019 se verificó la relación de las calificaciones por ejes de formación. En la siguiente imagen se aprecia el promedio de notas obtenidas por los estudiantes en áreas relacionadas a los fundamentos del diseño, materias aplicadas, materias teóricas y genéricas; se puede apreciar que la tendencia lineal de las materias aplicadas, está por debajo de la línea de los Fundamentos de Diseño, lo cual indica que los alumnos están teniendo menores calificaciones. 
Figura 1

Calificaciones de los ejes de

formación y líneas de tendencia

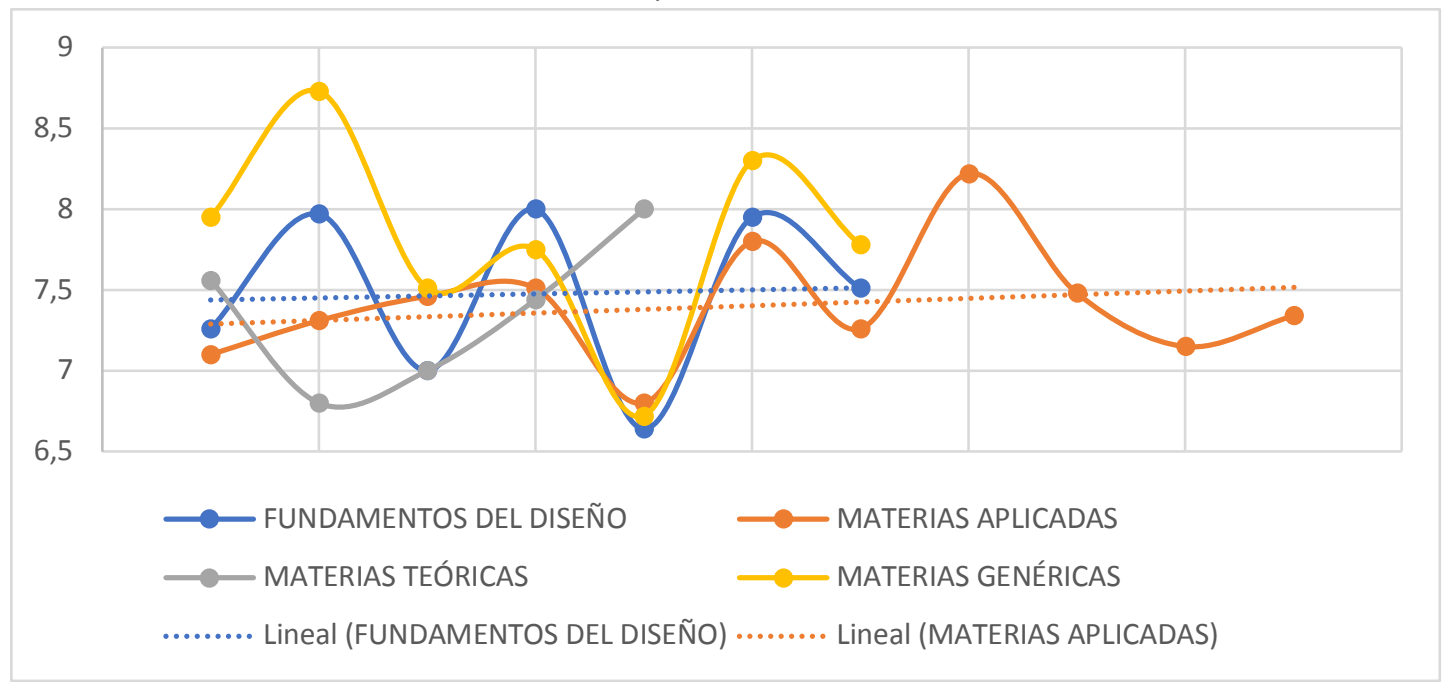

Estas cifras proyectaron la posibilidad de que existan problemas en la asimilación de los contenidos relacionados a los Fundamentos del diseño y contenidos teóricos de la carrera, que podrían estar influyendo en las asignaturas aplicadas y la calidad de los resultados de aprendizaje. En la siguiente imagen se muestra de manera comparativa los promedios por áreas de formación en la Carrera de Diseño de la Universidad Indoamérica Sede Quito.

Figura 2

Promedio de calificaciones en las distintas áreas de formación

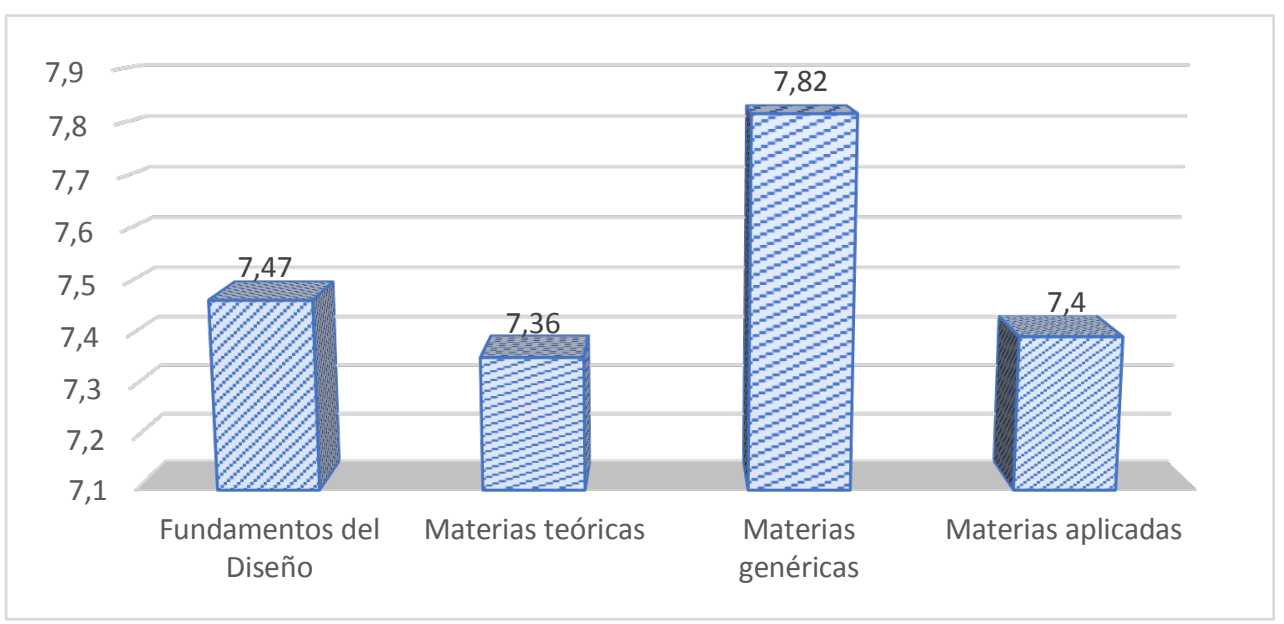

A partir del análisis de la gestión académica y las calificaciones en las distintas áreas de formación de la Carrera de Diseño de la Universidad Indoamérica, se plantea la ejecución de un estudio que pueda determinar los factores que generan falencias en las competencias relacionadas a la composición de piezas visuales, que se aprecia en las asignaturas aplicadas y que inciden en las calificaciones de los estudiantes. Con la finalidad de corroborar los datos se amplía el estudio a seis reconocidas Instituciones de Educación Superior que imparten las Carreras de Diseño en el Distrito Metropolitano de Quito. 


\subsection{Hacia un estudio más amplio}

El estudio de manera general pretendió establecer una conexión entre lo que el estudiante cree que conoce y lo que en realidad sabe en el sentido teórico y práctico; para realizar este trabajo se recurre a un estudio metodológico de tipo descriptivo (Fernández, Cordova, \& Cordero, 2002), con enfoque cualitativo y cuantitativo (Galeano, 2004), que emplea el método analítico - sintético, para interpretar datos obtenidos mediante la aplicación de entrevistas a profundidad (Torres, Paz, \& Salazar, 2019) a docentes de cuatro universidades, esta información se empleó para desarrollar un cuestionario que tuvo la validación de distintos docentes, especialistas en Diseño y en Educación de diversas instituciones de Educación Superior.

El instrumento fue aplicado en las Universidades: Indoamérica, Católica y De las Américas UDLA; además, de los Institutos Metro Design y el Instituto de Artes visuales de Quito IAVQ. Se aplicó el cálculo óptimo de muestra para poblaciones finitas sin varianza (Badii, Castillo, \& Guillen, 2017), se estableció un universo de 500 personas. Se aplicaron 200 encuestas considerando el número de estudiantes por cada institución, un margen de confianza del $95 \%$ y un error estimado de $5 \%$. En la figura 3 se aprecia la distribución de los estudiantes encuestados en la muestra por institución.

Figura 3

Composición de la muestra

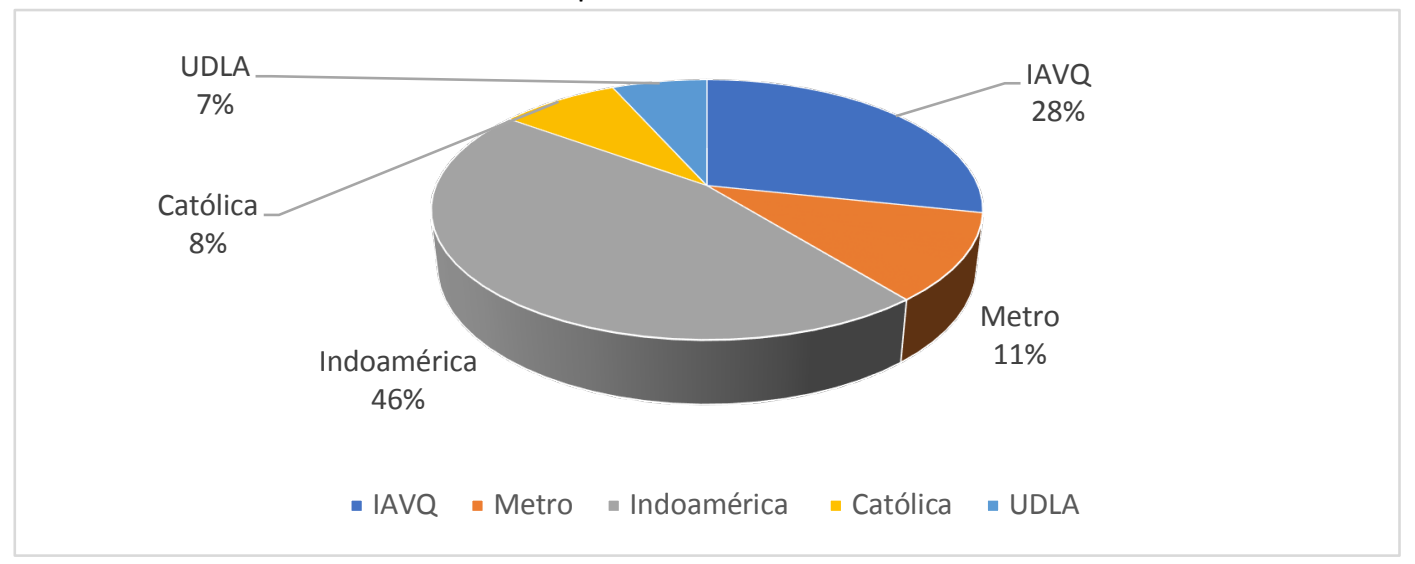

El instrumento permitió recoger los datos de distintos estudiantes que superaron el tercer semestre de sus respectivas carreras y que culminaron los periodos en los que se imparten las materias básicas. El cuestionario valida los conocimientos de los estudiantes en cinco de los aspectos más relevantes de los Fundamentos del Diseño, se abarcaron los temas de elementos del diseño, forma, composición, color y principios Gestalt.

En cada uno de los temas que se consultó a los estudiantes, se realizó una pregunta de escala numérica que permite medir la percepción sobre sus propios conocimientos; posteriormente, se realizaron preguntas cerradas de selección múltiple sobre aspectos teóricos y finalmente se le plantea un caso aplicativo. Se emplearon criterios contemporáneos para la recolección de datos (Vidal Diaz, 2000). El cuestionario se aplicó de manera digital mediante formularios de Google, los campos tenían validaciones específicas de contenido y se realizaron verificaciones de seguridad; se llegaron a descartar algunas encuestas debido a inconsistencias en los datos proporcionados, reajustando el nivel de confianza y el error conforme a lo descrito anteriormente. 


\section{Resultados}

\subsection{Percepción de conocimientos}

La investigación descriptiva permite emplear las distribuciones de probabilidad de variable continua (Llinás Solano \& Rojas Álvarez, 2005), para modelar fenómenos cuyas causas subyacentes corresponden a una gran cantidad de variables incontrolables y en las que otro tipo de representaciones resultarían confusas. La percepción de los estudiantes no puede reducirse a un cálculo de promedios, por lo que se decidió aplicar el modelo de intervalos de confianza, calculando la media y la desviación en cada tema (Castaño-Vergara, D. M., \& Cardona-Arango, D, 2015). La aplicación de la función gaussiana conforme se aprecia en la Figura 4, permitió establecer el intervalo en que los estudiantes valoraron su conocimiento.

Figura 4

Distribución normal en el nivel de percepción de cada tema

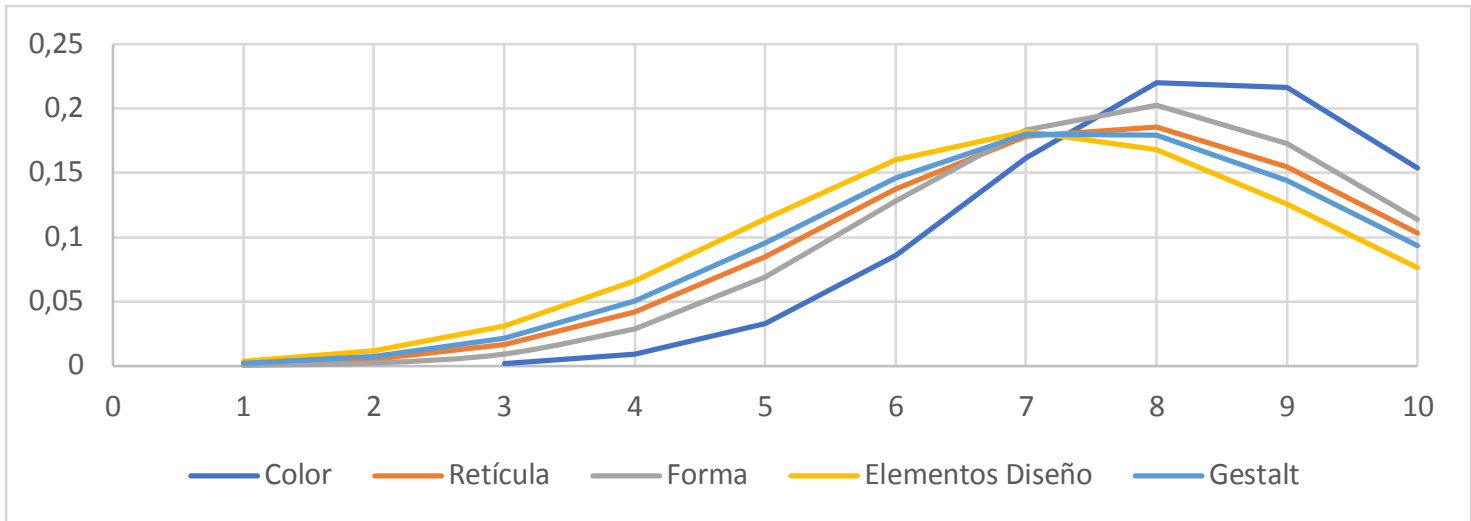

La tabla 1 presenta los rangos de desviación típica y confianza, la función de error se calculó con 5\%, por lo que se deduce que cualquier valor en el futuro estará dentro del comportamiento descrito.

Tabla 1

Intervalos de percepción de conocimiento de los estudiantes por fundamento

\begin{tabular}{|l|c|c|}
\hline Tema & \multicolumn{2}{|c|}{ Intervalo 1 - 10 } \\
\hline Teoría de color & 7.5 & 9.5 \\
\hline Principios Gestalt & 6.5 & 8.5 \\
\hline Retícula compositiva & 6.5 & 8.5 \\
\hline Elementos del Diseño - Forma & 5.5 & 8.5 \\
\hline
\end{tabular}

Por los datos obtenidos es posible señalar que el tema que los estudiantes creen conocer mejor es la teoría de color y el que consideran saber menos son los elementos del diseño, en donde se aprecia un intervalo de mayor amplitud y el límite superior más bajo.

\subsection{Resultados por cada fundamento del diseño}

En la tabla 2 se expresan los resultados de la encuesta, en términos de efectividad, tanto en las preguntas teóricas como prácticas. En cada uno de los fundamentos se aprecia una disminución de efectividad en la resolución de ejercicios prácticos de hasta el $30 \%$. 
Tabla 2

Intervalos de percepción de conocimiento de los estudiantes por fundamento

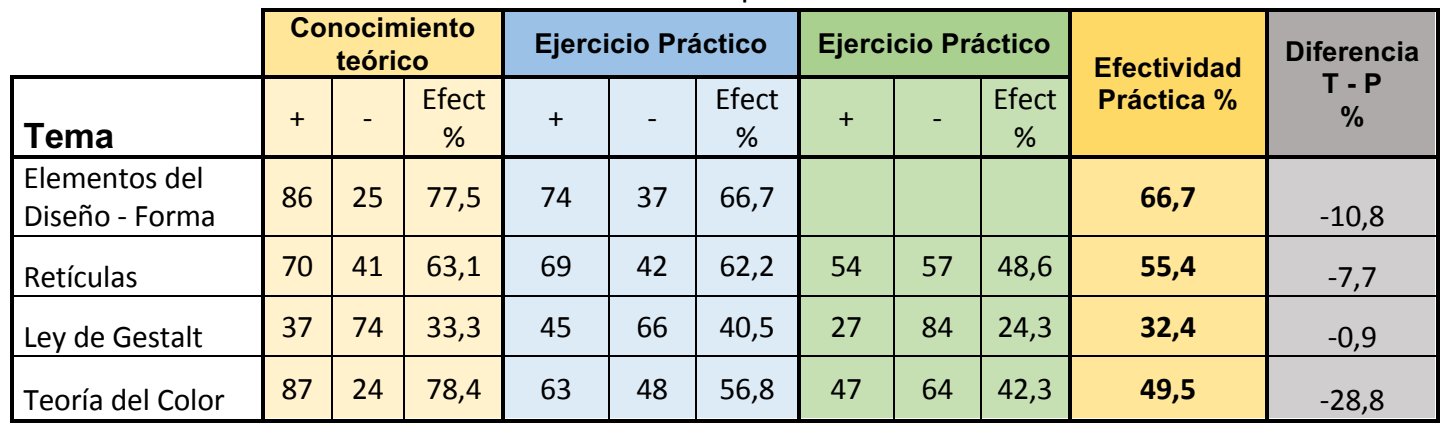

\section{Los elementos del diseño - forma}

Se les pidió a los estudiantes reconocer los elementos del diseño en un listado, acorde a la tabla 2 se registró $66,7 \%$ de resoluciones correctas; los resultados sugieren la existencia de confusión entre los elementos del diseño y conceptos relacionados a la forma. En este ámbito se puede apreciar una diferencia de $10 \%$ entre la valoración teórica y la efectividad para solucionar la práctica.

Como dato adicional, se pudo corroborar en una pregunta de control que el $82 \%$ de los estudiantes lograron identificar correctamente las formas propuestas en el instrumento. Se evidenció que un $13 \%$ de estudiantes estarían confundiendo la clasificación de la forma, con algunas de sus características.

\section{Retículas compositivas}

Se pudo apreciar que el $63.1 \%$ de los estudiantes conocen o tienen nociones sobre la utilidad de una retícula y su incidencia en una composición gráfica; pero, en la tabla 2 se aprecia que los encuestados confunden la utilidad de una retícula con conceptos relacionados a la aplicación de estructuras modulares repetitivas, algo que se refiere al ámbito del Diseño Tridimensional.

En el ejercicio práctico se pidió a los estudiantes reconocer las retículas que fueron empleadas en dos piezas publicitarias, en ambos intentos el instrumento registró un promedio de $55.4 \%$ de respuestas afirmativas, conforme se aprecia en la figura 5. Acorde a los especialistas consultados sobre estos resultados, esta deficiencia en la formación básica de los diseñadores comprometería sus competencias en el ámbito de la producción gráfica tanto de piezas publicitarias, editoriales y la producción de interfaces interactivas para distintos productos o dispositivos; así mismo explicaría en gran medida las deficiencias encontradas por los docentes en los resultados de aprendizaje de las materias aplicadas que se imparten en los niveles superiores.

Figura 5

Aciertos en pregunta de aplicación práctica sobre retículas compositivas

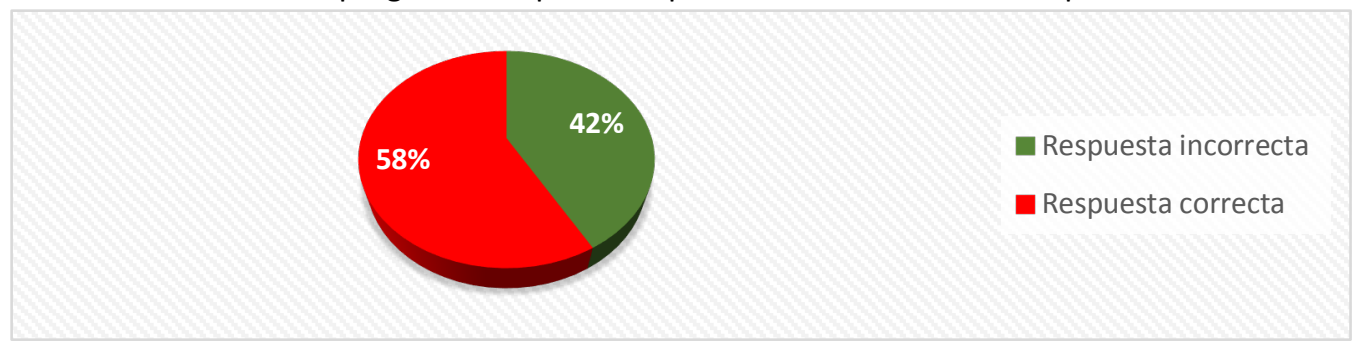

\section{Leyes de la Gestalt}

En la medición de estos principios básicos del diseño gráfico, se pudo determinar que existe una asimilación casi homogénea en la teoría y la práctica; sin embargo, esta asimilación es altamente deficiente, ya que el factor de efectividad promedio está por debajo del 33\%; esta cifra es preocupante ya que el conocimiento de estos 
fundamentos incide directamente en la conceptualización visual y la construcción del discurso que sustenta la interacción del diseño con el usuario.

\section{Teoría del color}

En las preguntas relacionadas a la teoría del color, se observa que son los conceptos que se encuentran menos asimilados a nivel práctico, la diferencia de efectividad entre la teoría y la práctica se encuentra en $28,8 \%$; es decir que, si bien los estudiantes pueden reconocer ejercicios de índole teórica, no logran aplicar esas nociones en la práctica. Respecto al color, se dio una particularidad, la pregunta de control práctica, era la misma que en la teoría se resolvió satisfactoriamente, pero se mostraba con distinta intensidad en los tonos; en general los estudiantes no lograron identificar la relación con la teoría y consecuentemente no pudieron resolver el ejercicio de manera correcta.

\section{Conclusiones}

El estudio realizado permitió determinar desde el contexto académico, que los estudiantes presentan deficiencias para vincular la teoría con la práctica, las cifras son claras respecto a, que la percepción de conocimiento que los estudiantes poseen, no concuerda con lo que saben y mucho menos con lo que demuestran conocer en un ejercicio aplicativo. Estas carencias han incidido en las calificaciones y los resultados de aprendizaje que son significativamente bajos en las asignaturas de la red profesionalizante.

La percepción de los estudiantes sobre sus conocimientos en la aplicación de los fundamentos del Diseño en otras asignaturas es incorrecta, es más alarmante pensar que este problema se podría estar trasladando al campo profesional, por lo que es necesario que se tomen medidas urgentes para revertir esta situación, reorientando distintos esfuerzos en beneficio de la construcción de las competencias de los estudiantes.

Acorde a la bibliografía revisada, las entrevistas realizadas a docentes y estudiantes, las causas de este problema subyacen en la necesidad de una mejor formación en estrategias pedagógicas para los docentes, un importante énfasis en la revisión de las actividades autónomas que los estudiantes realizan durante los primeros periodos de su formación profesional, una orientación adecuada de las prácticas de integración de saberes, el seguimiento y orientación en las prácticas pre-profesionales y los proyectos de vinculación. Otro factor que parecería incidir es el manejo de los recursos y material de apoyo que utilizan los docentes, quienes deberían usar recursos actuales, de preferencia digitales e interactivos, al parecer los materiales convencionales de carácter demostrativo, ya no estarían promoviendo adecuadamente el aprendizaje significativo, por lo que es conveniente que se realicen distintas reflexiones en base a los modelos pedagógicos con los que se está contextualizando los problemas de la profesión.

\section{Trabajo futuro}

Desde el ámbito del Diseño, se está proponiendo una reorientación de distintas estrategias pedagógica al proceso de enseñanza aprendizaje de los Fundamentos del Diseño, de manera que los recursos y esfuerzos de los distintos actores involucrados en la asimilación de estos conceptos trasciendan las materias básicas y reciban una retroalimentación constante a lo largo de toda la formación profesional; de la misma manera, se está reorganizando e incorporando nuevas tecnologías al material de apoyo educativo que emplean los docentes durante sus clases. Estas acciones irán paulatinamente generando datos que demuestren una mejor comprensión de las particularidades en la formación de artistas visuales y diseñadores gráficos. 


\section{Referencias}

Badii, Castillo, \& Guillen. (2017). Tamaño óptimo de la muestra. San Nicolás, Nuevo León, Mexico: Innovaciones de negocios. doi:1665-9627

Canelos, R. (2010). Formulación y Evaluación de un Plan Negocio. Quito, Ecuador: Universidad Internacional del Ecuador. doi:978-9942-03-111-2

Castaño-Vergara, D. M., \& Cardona-Arango, D. (2015). Percepción del estado de salud y factores asociados en adultos mayores. Revista de Salud Pública, 171-183.

Fernández , S., Cordova, A., \& Cordero , J. M. (2002). Estadística descriptiva. Madrid: Esic .

Galeano, M. E. (2004). Diseño de proyectos en la investigación cualitativa. Medellín, Colombia: Universidad EAFIT.

Llinás Solano, H., \& Rojas Álvarez, C. (2005). En Estadística descriptiva y distribuciones de probabilidad. Barranquilla, Colombia: Universidad del Norte.

Lupton, E., \& Phillips, J. C. (2009). Diseño Gráfico: Nuevos fundamentos. Barcelona: Ediciones Gustavo Gili.

Torres, M., Paz, K., \& Salazar, F. (2019). Métodos de recolección de datos para una investigación. Guatemala: Universidad Rafael Landivar.

Vidal Diaz, d. (2000). Utilización de nuevas tecnologías para el proceso de" recogida de datos" en la investigación social mediante encuesta. España: Revista Española de Investigaciones Sociológicas.

Wong, W. (1989). Fundamentos del Diseño Bi- y Tri-dimensional (6 ed.). Madrid, España: Gustavo Gili.

Esta obra está bajo una Licencia Creative Commons Attribución-NoCommercial 4.0 International

\section{(cc) EY-NC}

